\title{
Visualization of microRNA-21 Dynamics in Neuroblastoma Cells Using Magnetic Resonance Imaging Based on a microRNA-21-responsive Reporter Gene
}

\section{Guangcheng Bao}

Chongqing Medical University Affiliated Children's Hospital

Jun Sun

Chongqing Medical University Affiliated Children's Hospital

Jie Huang

Chongqing Medical University Affiliated Children's Hospital

\section{Helin Zheng}

Chongqing Medical University Pediatric College: Chongqing Medical University Affiliated Children's Hospital Jie Wei

Chongqing Medical University Pediatric College: Chongqing Medical University Affiliated Children's Hospital

\section{Yuanqiao Fu}

Chongqing Medical University Affiliated Children's Hospital

Jiawen Qiu

Chongqing Medical University Affiliated Children's Hospital

Jingxin Hou

The Second Affiliated Hospital of Chongqing Medical University

\section{Xuefeng Zou}

Guizhou Education University

\section{Bin Xiang}

Chongqing University

Jinhua Cai ( $\nabla$ cai_jinhua@126.com )

Department of Radiology, Children's Hospital of Chongqing Medical University https://orcid.org/0000-00017971-8936

\section{Research}

Keywords: microRNA-21, ferritin heavy chain, magnetic resonance imaging, neuroblastoma

Posted Date: July 8th, 2021

DOl: https://doi.org/10.21203/rs.3.rs-678209/v1 
License: (우 This work is licensed under a Creative Commons Attribution 4.0 International License. Read Full License 


\section{Abstract}

Background: MicroRNAs (miRs) have been shown to be closely associated with the occurrence and development of tumors and to have potential as diagnostic and therapeutic targets. The detection and quantification of miRs by noninvasive imaging technology is crucial for deeply understanding their biological functions. Our aim was to develop a novel miR-21-responsive gene reporter system for magnetic resonance imaging (MRI) visualization of the miR-21 dynamics in neuroblastoma.

Methods: The reporter gene ferritin heavy chain (FTH1) was modified by the addition of 3 copies of the sequence completely complementary to miR-21 (3xC_miR-21) to its 3'-untranslated region (3' UTR) and transduced into SK$\mathrm{N}-\mathrm{SH}$ cells to obtain SK-N-SH/FTH1-3xC_miR-21 cells. Then, the antagomiR-21 was delivered into cells by graphene oxide functionalized with polyethylene glycol and dendrimer. Before and after antagomiR-21 delivery, FTH1 expression, MRI contrast and intracellular iron uptake were assayed in vitro and in vivo.

Results: In the SK-N-SH/FTH1-3xC_miR-21 cells, FTH1 expression was in an "off" state due to the combination of intratumoral miR-21 with the 3 ' UTR of the reporter gene. AntagomiR-21 delivered into the cells bound to miR21 and thereby released it from the 3' UTR of the reporter gene, thus "switching on" FTH1 expression in a dosedependent manner. This phenomenon resulted in intracellular iron accumulation and allowed MRI detection in vitro and in vivo.

Conclusions: MRI based on the miR-21-responsive gene reporter may be a potential method for visualization of the endogenous miR-21 dynamics.

\section{Background}

MicroRNAs (miRs) are endogenous single-stranded small-molecule RNAs that are approximately 20-25 nucleotides long, do not encode proteins, and have a variety of important regulatory functions in cells [1]. miRs can target and bind the 3'-untranslated regions (3' UTRs) of specific messenger RNAs (mRNAs) and induce their translational repression or degradation, thereby decreasing the expression of proteins encoded by the targeted mRNAs. This novel mode of posttranscriptional gene regulation plays an important role in extensive biological processes, such as cell growth, differentiation, apoptosis, tumorigenicity and chemoresistance $[2,3]$.

Since the first report of the role of miRs in oncogenesis [4], many miRs involved in cancer development have been identified. Among these, some function as onco-miRs by promoting tumor proliferation and angiogenesis, while some are associated with tumor inhibition [5-7]. The different functions of these miRs may be related to the type and stage of cancer development. Currently, the expression profiles of miRs and their potential targets have been widely studied in breast [8], lung [9], prostate [10], colon [11], gastric [12] and ovarian [13] cancer. Among various cancer-associated miRs, miR-21 has attracted substantial attention as a potential diagnostic and therapeutic cancer target since it has been reported to be the only carcinogenic miR that is overexpressed in several types of solid tumors, including neuroblastoma, glioblastoma, breast cancer, colorectal cancer and prostate cancer $[4,8,10,11,14]$. On the other hand, a chemically modified antisense RNA oligonucleotide (antagomir-21) has been used to target and repress the activity of miR-21, thereby inhibiting tumor development $[15,16]$. 
Considering the potential of miR to serve as a diagnostic and therapeutic biomarker in cancer, investigations of the biological properties and expression patterns of miRs are crucial. The conventional methods for detecting endogenous miRs include northern blotting [17], quantitative reverse-transcriptase polymerase chain reaction (qRT-PCR) [9], small RNA sequencing [18] and microarray [19]. However, these methods are invasive, time consuming and nonreproducible. In addition, they require cell lysis and are not suitable for detecting miR expression in living cells. Therefore, the development of noninvasive methods is imperative for elucidating the expression and regulation of miRs in vivo.

At present, several noninvasive imaging techniques, such as optical imaging, radionuclide imaging and magnetic resonance imaging (MRI), have been applied to detect biological processes in vivo at the cellular or molecular level $[20,21][20,21]$. Of these imaging methods, MRI is thought to be ideal due to its advantages, including deep tissue penetration, high tissue resolution, repeatable examination and nonrequirement for radiation. Molecular MRI usually relies on a reporter gene, which can be integrated into the cellular genome and allows for the long-term monitoring of biological events. Among various MRI reporter genes, ferritin heavy chain 1 (FTH1) has attracted substantial attention because its expression can induce the effective cellular uptake of endogenous iron, which leads to sensitive MRI signal contrast [22-28][22-28]. In our previous studies, we successfully monitored mesenchymal stem cells with FTH1-based MRI in vitro and in vivo [24, 29][24, 29]. Considering the advantages of FTH1 as an endogenous reporter gene, it is worth investigating the feasibility of FTH1-based MRI to visualize miR activities, and this method could serve as a noninvasive imaging modality for miR detection.

In this study, we designed a novel miR-21-responsive FTH1 expression system in which the reporter gene FTH1 was modified by the addition of 3 copies of the sequence completely complementary to miR-21 (3xC_miR-21) to its $3^{\prime}$ UTR. When the reporter gene system was transduced into neuroblastoma cells, in which miR-21 functions as an oncogenic biomarker, its expression was expected to be switched "off" due to the combination of intratumoral miR-21 with the 3' UTR of the reporter gene. An exogenous antisense oligonucleotide (antagomiR21) was then delivered into the cells and expected to bind with miR-21 in a competing manner, thereby releasing miR-21 from the FTH1 3' UTR and switching "on" the reporter gene expression (Fig. 1). With the reporter gene system, we aimed to provide a noninvasive and efficient imaging modality for visualizing the activity of miR-21 and its response to gene therapy.

\section{Methods}

\subsection{Construction of the miR-21-responsive FTH1 expression vector}

The CDNA of human FTH1 (Gene ID: 2495) was obtained via polymerase chain reaction (PCR) amplification using the following primers: forward, AACCGTCAGATCGCACCGGTGCCACCATGACGACCGCGTCCACCTC and reverse, TCCTTGTAGTCCATGAATTCGCTTTCATTATCACTGTCTC. The synthesized FTH1 cDNA was subcloned into the EcoR I and Sma I restriction endonuclease sites of the plasmid pLVX-mCMV-ZsGreen1-Puro to obtain the recombinant plasmid vector pLVX-mCMV-ZsGreen1-Puro-FTH1 (pLV-FTH1). Then, the DNA oligonucleotides containing 3xC_miR-21 (sense 5'

GTAGCTTATCAGACTGATGTTGATAGTAGTAGCTTATCAGACTGATGTTGATAGTAGTAGCTTATCAGACTGATGTTGA 
3'; antisense 5'

TCAACATCAGTCTGATAAGCTACTACTATCAACATCAGTCTGATAAGCTACTACTATCAACATCAGTCTGATAAGCTAC

$\left.3^{\prime}\right)$ were synthesized and ligated into pLV-FTH1 to yield the final lentiviral vector plasmid containing 3xC_miR-21 (pLV-FTH1-3xC_miR-21). After verification by PCR analysis and DNA sequencing, the plasmid pLV-FTH13xC_miR-21 was cotransfected together with psPAX2 and pMD2.G into 293 T packaging cells (Invitrogen, Carlsbad, CA, USA) to produce the lentivirus containing FTH1-3xC_miR-21.

\subsection{Cell culture and establishment of stable transgenic cells}

The wild-type human neuroblastoma cell line (SK-N-SH/WT) was provided by the Chongqing Institute of Pediatric Medicine Research and cultured in Dulbecco's modified Eagle's medium (DMEM; Gibco, Grand Island, NY, USA) containing 10\% fetal bovine serum (FBS; Gibco, Grand Island, NY, USA) and $0.5 \%$ streptomycin at $37^{\circ} \mathrm{C}$ in a moist environment containing 5\% CO2. According to the lentivirus transfection protocol, cells at $30-40 \%$ confluence were transfected with the FTH1-3xC_miR-21 lentivirus and screened with $10 \mu \mathrm{g} / \mathrm{ml}$ puromycin. Finally, the surviving clones were isolated and named SK-N-SH/FTH1-3xC_miR-21 cells, with SK-N-SH/WT cells and SK-N-SH/FTH1 (without 3xC_miR-21) cells serving as controls.

\subsection{Assessment of cell growth}

To investigate the effect of gene transduction on cell viability, cell growth was assayed by Cell Counting Kit-8 (CCK-8) colorimetry (Beyotime, Nanjing, Jiangsu, China). SK-N-SH/WT, SK-N-SH/FTH1 and SK-N-SH/FTH1$3 \times$ C_miR-21 cells were cultured in 96-well plates for $72 \mathrm{~h}$, and the relative changes in the three cell lines were determined.

\subsection{AntagomiR-21 transfection into cells}

The antisense RNA oligonucleotide, antagomiR-21, was provided by GenePharma, Shanghai, China. The sequence of antagomiR-21 was as follows: 5'-GUCAACAUCAGUCUG AUA AGCUA-3.

For improvement of the transfection efficiency of antagomiR-21, graphene oxide (GO) modified with polyethylene glycol (PEG) and polyamidoamine dendrimer (GO-PEG-dendrimer) was used as gene delivery carrier. GO was synthesized by previously reported methods [30-35], and the detailed protocol for synthesizing GO-PEG-dendrimer and its characterization are shown in the supporting document.

To explore the optimal structure of GO-PEG-dendrimer for efficiently delivering anagomiR-21 into SK-N-SH cells, we mixed GO-PEG-dendrimer at GO/PEG molar ratios of 5:1, 1:5 and 1:1 with 30 pmol of anagomiR-21 modified by the fluorescence dye Cy3 (anagomiR-21-Cy3) to prepare GO-PEG-dendrimer/anagomiR-21-Cy3 complexes. Then, $2 \mu \mathrm{L}$ of siRNA-Mate (GenePharma, Shanghai, China), a commonly used small-molecule RNA transfection agent, was mixed with 30 pmol of antagomiR-21-Cy3 to obtain the siRNA-Mate/antagomiR-21-Cy3 complex as a control. The obtained complexes were incubated with SK-N-SH cells for 6 h, washed 3 times, fixed with $4 \%$ formaldehyde and stained with 4',6-diamidino-2-phenylindole (DAPI). Fluorescence images were obtained by a fluorescence microscope (Nikon A1R, Tokyo, Japan).

A CCK-8 assay was used to compare the cytotoxicity of the GO-PEG-dendrimer complexes and siRNA-Mate. SK$\mathrm{N}$-SH cells were divided into two groups, cultured in 96-well plates at a density of $6 \times 10^{3}$ cells per well, and cocultured with the GO-PEG-dendrimer (GO/PEG ratio 1:1) or siRNA-Mate at equal concentrations for $72 \mathrm{~h}$. Then, the absorbance was measured every $12 \mathrm{~h}$ after the addition of CCK-8 reagent. 


\subsection{Western blot and qRT-PCR assays}

FTH1 expression in SK-N-SH/WT, SK-N-SH/FTH1 and SK-N-SH/FTH1-3×C_miR-21 cells was detected by qRTPCR. Briefly, total RNA was extracted using a TaKaRa MiniBEST Universal RNA Extraction Kit (TaKaRa®, Kyoto, Japan) and then reverse transcribed into cDNA using HiScript ${ }^{\circledR}$ II Q RT SuperMix (Vazyme, Nanjing, China) for qPCR, which was carried out using AceQ ${ }^{\circledR}$ qPCR SYBR Green Master Mix (Vazyme) on an AB Step One plus Real Time PCR System (Applied Biosystems AB, Waltham Mass, USA). GAPDH expression served as the quantitative internal control for FTH1, and each experiment was performed in triplicate. The primers for GAPDH and FTH1 detection were as follows: GAPDH-F AGAAGGCTGGGGCTCATTTG and GAPDH-R AGGGGCCATCCACAGTCTTC; FTH1-F GGAATTCATGACGACCGCGTCCAC and FTH1-R CCCCGGGAGCTTTCATTATCACTGTCTCCC.

Simultaneously, proteins were extracted from the three cell lines and prepared for Western blot (WB) analysis. Briefly, the cells were cleaved in cleavage buffer (Sigma, St. Louis, Missouri, USA) containing protease inhibitors, phosphatase inhibitors and $100 \mathrm{mM}$ benzene sulfonyl fluoride, and the total protein concentration in the sample was determined by the BCA method. Thirty micrograms of protein from each sample was then added to a $12 \%$ sodium dodecyl sulfate-polyacrylamide gel and transferred onto a polyvinylidene fluoride (PVDF) membrane (Bio-Rad, California, USA). For detection of FTH1, the membrane was blocked with $5 \%$ bovine serum albumin (BSA; Beyotime) for $1 \mathrm{~h}$ and then incubated with a primary antibody that specifically recognized FTH1 (rabbit anti-FTH1, 1:1000; Abcam, Cambridge, England) or GAPDH (rabbit anti-FTH1, Abcam) overnight at $4^{\circ} \mathrm{C}$. The membrane was then washed with Tris-buffered saline containing Tween-20 and incubated with a secondary antibody (goat anti-rabbit IgG, 1:5000; Sigma) for $2 \mathrm{~h}$. The protein bands were observed using an enhanced chemiluminescence kit (Sigma). The relative expression of FTH1 in SK-N-SH/FTH1-3C×_miR-21 cells was normalized to that of GAPDH, and semiquantitative analysis was carried out based on the band strength.

For determination of the correlation between FTH1 expression and the amount of antagomiR-21, SK-N-SH/FTH1$3 \times C \_m i R-21$ cells transfected with antagomiR-21 at different concentrations $(0,10,10,20,30,40,40,50$, or 60 $\mathrm{nmol} / \mathrm{L}$ ) were cultured for $24 \mathrm{~h}$, and cellular proteins were collected and prepared for WB analysis.

\subsection{MRI of cell pellets}

To assess the effect of FTH1 expression on cellular iron transfer, we cultured SK-N-SH/FTH1-3C×_miR-21 cells in medium containing $500 \mu \mathrm{mol} / \mathrm{L}$ ferric ammonium citrate (FAC; Sigma) and then transfected them with antagomiR-21 at the same concentration gradient as that used for the WB experiment. After $24 \mathrm{~h}$, all the treated cells were washed thoroughly with phosphate-buffered saline (PBS) to remove the free FAC, digested with ethylenediaminetetraacetic acid (EDTA), suspended in PBS, and then transferred into a 600-inch I-Eppendorf tube to prepare the cell phantom for MRI in vitro. The cellular phantom was imaged using a $7.0 \mathrm{~T}$ MR scanner (Bruker, Karlsruhe, Germany). The spin echo (SE) T2 weighted imaging (T2WI) parameters were as follows: time of repetition (TR), 2,500 ms; time of echo (TE), $35 \mathrm{~ms}$; field of view (FOV), $120 \mathrm{~mm} \times 120 \mathrm{~mm}$; slice thickness, $1 \mathrm{~mm}$; slice interval, $0.1 \mathrm{~mm}$. The parameters for the multiecho sequence were as follows: TR, 2,000 ms; TE, $8 \sim 200 \mathrm{~ms}$ with a step size of $8 \mathrm{~ms}$ (25-point T2 mapping); other parameters, including the FOV, matrix and slice thickness, matched those used for T2WI imaging. T2 maps were obtained by image postprocessing, and the R2 value was measured.

To evaluate FTH1 expression and its effect on iron transfer in groups of cells treated with or without antagomir21, SK-N-SH/WT, SK-N-SH/FTH1 and SK-N-SH/FTH1-3×C_miR-21 cells were cultured with antagomir-21 at the 
optimal concentration and $500 \mu \mathrm{mol} / \mathrm{L}$ FAC for $24 \mathrm{~h}$. Before and after antagomiR-21 treatment, the three cell lines were subjected to MRI examination.

\subsection{Intracellular iron detection and quantification}

Prussian blue staining and transmission electron microscopy (TEM) were performed to detect intracellular iron accumulation. SK-N-SH/WT, SK-N-SH/FTH1 and SK-N-SH/FTH1-3×C_miR-21 cells transfected with or without antagomir-21 were cultured together with $500 \mu \mathrm{mol} / \mathrm{L}$ FAC for $24 \mathrm{~h}$. Then, the cells were harvested and subjected to Prussian blue staining and TEM examination according to our previous protocols [24]. The intracellular accumulation of iron was observed under a light microscope (Nikon, Tokyo, Japan) or an H-7500 transmission electron microscope (Hitachi, Tokyo, Japan). For quantification of the intracellular iron content, $1 \times 10^{6}$ cells per group were prepared according to the previous protocols [36]. The iron concentration was measured using an atomic absorption spectrophotometer (Huaguang HG-960 2A, Shenyang, China). Each sample was measured 3 times. The concentration values are presented in units of $\mathrm{pg} /$ cell.

\subsection{In vivo experiments}

The experimental animals were purchased from the Department of Medical Experimental Animals of Chongqing Medical University and raised at the Experimental Animal Center of our hospital. All animal protocols used in this study were reviewed and approved by the Animal Care and Use Committee of Chongqing Medical University, and the experimental procedures were performed in accordance with the National Institutes of Health guidelines. All efforts were made to minimize animal suffering.

The nude mice were divided into the following 3 groups: SK-N-SH/WT, SK-N-SH/FTH1 and SK-N-SH/FTH1$3 \mathrm{C} \times$ _miR-21. A total of $2 \times 10^{6}$ cells were inoculated subcutaneously into the right flanks of nude mice in each of the three groups to establish the transplanted tumor model of neuroblastoma. To enhance iron recruitment into cell xenografts, the animals were treated with FAC at a dose of $5 \mathrm{mg} / \mathrm{L}$ via their drinking water. When the tumors reached approximately $300 \mathrm{~mm}^{3}$ in size, the GO-PEG dendrimer/antagomiR-21 complex was administered via caudal vein injection at an antagomiR-21 dose of $30 \mathrm{nmol} / \mathrm{g}$ according to the manufacturer's instructions.

Before and $24 \mathrm{~h}$ after antagomiR-21 treatment, the animals were anesthetized and subjected to MRI examination. A 7.0 T MRI scanner was applied, and MR images were obtained by using the T2WI and multiecho sequences. The T2WI scanning parameters were as follows: TR, 2,500 ms; TE, $35 \mathrm{~ms}$. The multiecho parameters were as follows: TR, 2,000 ms; TE, $8 \sim 200$ ms with a step size of 8 ms (25-point T2 mapping); matrix, 380×300; FOV, $160 \mathrm{~mm}$; and slice thickness, $1.2 \mathrm{~mm}$. The R2 value was measured on T2 maps obtained by image postprocessing.

Immediately after MRI, the animals were sacrificed, and the masses were removed for histological examination. Prussian blue staining was also performed to detect iron accumulation in the tumors. In addition, the intratumoral iron content was quantified according to the protocols used for intracellular iron quantification and are presented in units of $\mathrm{mg} / \mathrm{g}$.

\subsection{Statistical analysis}

All data are expressed as the mean \pm standard deviation. Statistical Package for the Social Sciences version 13.0 (SPSS Inc., Chicago, IL, USA) was used for the statistical analyses. One-way analysis of variance and the least 
significant difference method were used to compare differences among the groups. $P$ values less than 0.05 were considered statistically significant.

\section{Results}

\subsection{The miR-21-responsive FTH1 reporter gene system and its effect on cell viability}

The miR-21-responsive FTH1 gene reporter system, namely, FTH1-3×C_miR-21, which contained 3 copies of a sequence completely complementary to miR-21 at the 3' UTR of the FTH1 gene, was successfully constructed. This reporter gene system was transduced into SK-N-SH cells, and SK-N-SH/FTH1-3×C_miR-21 cells were established.

To verify the activity of the reporter gene system, we evaluated FTH1 expression in the SK-N-SH/WT, SK-NSH/FTH1 and SK-N-SH/FTH1-3×C_miR-21 cells by qRT-PCR and WB. The qRT-PCR results showed that the mRNA expression levels of the FTH1 gene in the SK-N-SH/FTH1 and SK-N-SH/FTH1-3×C_miR-21 groups were similar and both were significantly higher than that in SK-N-SH/ WT group $(P<0.0001)$. The WB results showed that the protein level of FTH1 was increased significantly $(P<0.0001)$ in the SK-N-SH/FTH1 group but not altered significantly in the SK-N-SH/FTH1-3×C_miR-21 group compared with the SK-N-SH/WT group (Fig. 2a and b). These results indicated that miR-21 inhibited FTH1 expression at the post-transcriptional level.

To assess the safety of the reporter gene system, cell viability was evaluated. Morphologically, no changes were observed among SK-N-SH/WT, SK-N-SH/FTH1 and SK-N-SH/FTH1-3×C_miR-21 cells (Fig. 2c). The CCK-8 assay results also showed no significant differences in cell proliferation among the three groups (Fig. 2d).

\subsection{Gene transfection efficiency and cytotoxicity of the GO-PEG dendrimer}

Immunofluorescence staining and fluorescence intensity quantification showed that the GO-PEG dendrimer with various GO/PEG ratios differentially introduced antagomir-21 into cells. Among the three structures with GO/PEG ratios of $1: 5,1: 1$ and 5:1, the GO-PEG dendrimer with a GO/PEG ratio of 1:1 was the most efficient and was more efficient than the commonly used transfection reagent siRNA-Mate (Fig. 3a and b).

To study the cytotoxicity of the GO-PEG-dendrimer, we evaluated the survival rate of SK-N-SH cells. As shown in Fig. 3c, the cytotoxicity of the GO-PEG-dendrimer was much lower than that of siRNA-Mate, indicating that GOPEG-dendrimer is safe as a gene delivery vector.

\subsection{Dose-dependent FTH1 expression regulated by antagomiR- 21}

To investigate the ability of antagomiR-21 to knock down miR-21 and reverse FTH1 expression in SK-NSH/FTH1-3×C_miR-21 cells, we carried out WB to evaluate FTH1 expression in the cells treated with different concentrations of antagomir-21. AntagomiR-21 delivery into cells reversed FTH1 expression in a dose-dependent manner. In the absence of antagomiR-21, no obvious FTH1 expression was detected in the SK-N-SH/FTH13×C_miR-21 cells in which miR-21 suppressed the reporter gene by binding with the completely complimentary 
sequence of 3×C_miR-21. After transfection with antagomir-21, FTH1 expression increased gradually as the antagomir-21 concentration increased, peaking at $40 \mathrm{nmol} / \mathrm{L}$ antagomir-21, and then slightly decreased as the antagomiR-21 continuously increased (Fig. 4a and b). The cellular MRI T2WI and R2 measurements of cells treated with different concentrations of antagomiR-21 were consistent with the WB results (Fig. 4c-e).

\subsection{Cellular MRI contrast produced by the regulatory expression of FTH1}

The expression of FTH1 may increase the ability of cells to internalize and store iron ions, thereby decreasing the MRI signal. To assess the feasibility of using MRI to detect miR-21 activity regulated by antagomiR-21, we first determined the optimal concentration $(40 \mathrm{nmol} / \mathrm{L})$ at which antagomiR-21 induced the most obvious MRI signal change. Then, MRI was performed in 3 groups of cells treated with the optimal concentration of antagomiR-21: SK-N-SH/WT, SK-N-SH/FTH1 and SK-N-SH/FTH1-3×C_miR-21 cells. A significant signal reduction was observed in only the SK-N-SH/FTH1-3×C_miR-21 cells after antagomiR-21 transfection compared with the signal prior to antagomiR-21 treatment $(P<0.05)$. There were no changes in the MRI signals change before and after antagomiR-21 treatment in the other two groups of cells (Fig. 5a and b).

The Prussian blue staining and TEM results were consistent with the MRI findings. Before transfection of antagomir-21, large amounts of iron particles were detected in the cytoplasms of only SK-N-SH/FTH1 cells, while no iron accumulation was observed in SK-N-SH/WT or SK-N-SH/FTH1-3×C_miR-21 cells. After antagomiR-21 treatment, SK-N-SH/FTH1-3×C_miR-21 cells exhibited obvious iron accumulation compared with that prior to antagomiR-21 treatment. In the other two groups of cells, however, there was no change in iron accumulation before and after antagomiR-21 treatment (Fig. $5 \mathrm{c}$ and d). Quantification of the intracellular iron content (Fig. 5e) showed that the iron content in the SK-N-SH/FTH1-3×C_miR-21 cells was $0.96 \pm 0.17 \mathrm{pg} /$ cell after antagomiR-21 treatment, significantly higher than that before antagomiR-21 treatment $(0.12 \pm 0.02 \mathrm{pg} / \mathrm{cell})(P<0.05)$. There was no difference of iron content before and after antagomiR-21 treatment in the other two groups.

The above results suggest that miR-21 in SK-N-SH/FTH1-3×C_miR-21 cells can be inhibited by antagomiR-21 and reboot the expression of $\mathrm{FTH} 1$, thereby resulting in intracellular iron accumulation. This process can be detected by MRI signal changes.

\subsection{In vivo MRI of antigomiR-21 delivery}

Animal tumor models of three SK-N-SH cell lines were successfully established. Before administration of the GOPEG-dendrimer/antagomiR-21 complex, the MRI signal was obviously decreased and the R2 value was significantly increased in the SK-N-SH/FTH1 group $(P<0.0001)$ compared with the SK-N-SH/WT group, while those in the SK-N-SH/FTH1-3×C_miR-21 group did not differ from those in the SK-N-SH/WT group. After treatment with the complex, the SK-N-SH/FTH1-3×C_miR-21 group showed a significantly decreased MRI signal and increased R2 value compared with those prior to treatment $(P<0.05)$. In the other two groups, the MRI signals and R2 values did not differ before and after antagomiR-21 treatment (Fig. 6a and b).

Further intratumoral iron detection and quantification matched the MRI results. Intratumoral iron content was significantly increased after antagomiR-21 treatment in only the SK-N-SH/FTH1-3×C_miR-21 group compared with that prior to treatment (Fig. 6c and d). 


\section{Discussion}

Increasing evidence has demonstrated that miRs are involved in tumorigenesis and tumor progression in vitro and in vivo, which provides a new pathway for exploring the potential of miRs as biomarkers for tumor diagnosis and/or therapy $[2,3,15,37]$. In this study, we designed a novel gene reporter system which contained three copies of the sequence completely complimentary to miR-21 in the 3' UTR of FTH1 for the MRI visualization of miR-21 in neuroblastoma. The results showed that the miR-21-responsive reporter gene was sensitively regulated by miR-21 in neuroblastoma cells. Compared with the regular FTH1 reporter gene, the miR-21-responsive reporter gene was "switched off" by the combination of intratumoral miR-21 and the 3' UTR of FTH1. AntagomiR-21 induced exogenously "switched on" FTH1 expression in the cells by binding to miR-21 and thereby promoting its release from the FTH1 3' UTR. FTH1 expression induced a sufficient amount of intracellular iron accumulation for visualization by MRI in vitro and in vivo. In addition, the reporter gene expression reversed by antagomiR-21 was dose-dependent, suggesting that miR-21 can be quantified by MRI. To the best of our knowledge, this is the first report of using an MRI-based gene reporter system to study miR-21 dynamics in tumors. Our research provides a potential noninvasive imaging method for evaluating miR-21 as a diagnostic or therapeutic target.

Recently, optical imaging techniques based on luciferase or bioluminescent reporter genes have been applied in the miR research field and been shown to serve as noninvasive methods for detecting and quantifying miRs in vivo. Ko et al. [38] developed a reporter gene system, CMV/Gluc/3×PT-miR-124, and transduced it into stem cells, and the results showed miR-124a-dependent Gluc reporter expression during neural differentiation. Kim et al. [39] utilized a CMV/Gluc-3xPT_miR221 reporter system to image miR-221 in papillary thyroid carcinoma and showed that Gluc activity was regulated according to the miR-221 levels in vitro and in vivo. These optical imaging reporter gene systems have greatly expanded our understanding of miR activities in vivo. However, optical imaging modalities can be used only for studies in small animals or surface tissues due to their poor tissue penetration, which has limited their clinical application. In the present study, we utilized MRI to detect miR-21 activity and achieved results consistent with those of optical imaging studies. In addition, compared with optical imaging, MRI can better penetrate deep tissue, which may make it more applicable in the miR imaging research field and more feasible for clinical translation.

Employing antisense oligonucleotides to antagonize the functions of target miRs in vivo has been proposed as a potential gene therapy for various diseases [40-42]. This therapeutic strategy, however, has largely been impeded due to the lack of effective gene delivery carriers. In this study, we constructed a polyamidoamine dendrimer and PEG-functionalized GO conjugate for the delivery of antagomiR-21 into SK-N-SH cells. The efficiency of the GO-PEG-dendrimer to deliver antagomiR-21 was affected by the GO/PEG ratio. Among the three GO/PEG ratio groups, the GO-PEG dendrimer with a GO/PEG ratio of 1:1 delivered antagomiR-21 more efficiently than the commonly used transfection agent siRNA-Mate. The other two structures, which had GO/PEG ratios of $5: 1$ and 1:5, had delivery efficiencies that were similar to that of siRNA-Mate. We speculate that the decreased transfection efficiency of the GO-PEG-dendrimer with a GO/PEG ratio of 5:1 was due to the decreased solubility of GO-PEG dendrimers. The lower transfection efficiency of the GO-PEG-dendrimer with a GO/PEG ratio of 1:5 may be explained by the difficulty of the GO-PEG-dendrimer in entering the cell due to the increased molecular weight of PEG. Based on the optimal GO/PEG ratio, we further compared the cytotoxicities of GO-PEG-dendrimer and siRNA-Mate. The cell viability of the SK-N-SH cells transfected with the GO-PEG-dendrimer was higher than that of the cells transfected with siRNA-Mate during $72 \mathrm{~h}$ of continuous culture, suggesting that the GO-PEGdendrimer is a safe gene delivery vector. In addition, the in vitro and in vivo experiments revealed that the 
antagomiR-21 delivered by the GO-PEG-dendrimer definitely induced FTH1 expression by antagonizing miR-21, thereby indirectly confirming the GO-PEG-dendrimer efficiency as a gene delivery vector.

Although the miR-21-responsive FTH1 gene reporter system was proven to have potential for the MRI assessment of miR-21 function in tumor cells, there are several drawbacks in this study. First, the reporter gene system was transduced into the tumor cells in advance to evaluate the activity of miR-21, and this method is limited to experimental studies and is not feasible for clinical applications. To use this gene reporter system to detect intracellular miR-21 for diagnostic purposes in humans, the system should be delivered into tumor cells by an efficient gene carrier to examine the results in real time. Second, to increase reporter gene expression-induced intracellular iron uptake to a level that is sufficient to generate MRI contrast in vivo, exogenous iron was administered to the animal at a certain concentration. Further studies are needed to explore whether intracellular iron recruitment depends merely on endogenous iron concentrations and can lead to detectable MRI contrast. Third, the MRI examination was performed only once after antagomir-21 delivery in vivo in consideration of animal welfare and fast tumor necrotization. A more suitable tumor xenograft model should be established to allow MRI examinations at more time points, which may enable a longitudinal MRI monitoring study of miR functions.

In summary, we herein developed a novel miR-21-responsive gene reporter system for the MRI visualization of miR-21 dynamics in neuroblastoma. In the presence of active miR-21 in tumor cells, the reporter gene expression was in an "off" state due to the specific combination of miR-21 with 3 copies of completely complimentary sequences (3xC_miR-21) at the 3' UTR of the reporter gene. The exogenous delivery of antagomiR-21 into the cells by the GO-PEG-dendrimer switched "on" the reporter gene expression because miR-21 bound with antagomiR-21 in a competitive manner and was thus released from the FTH1 3' UTR. Reporter gene expression resulted in intracellular iron accumulation, thereby allowing MRI visualization. This study provides a noninvasive and feasible imaging method for the detection and quantification of miR-21, which is regarded as a potential diagnostic and/or therapeutic target in tumors.

\section{Abbreviations}

miRs: MicroRNAs; 3' UTRs: 3'-untranslated regions; mRNAs: messenger RNAs; qRT-PCR: quantitative reversetranscriptase polymerase chain reaction; MRI: Magnetic resonance imaging; FTH1: Ferritin heavy chain 1; 3xC_miR-21: 3 copies of the sequence completely complementary to miR-21; LV: Lentiviral vector; PCR: Polymerase chain reaction; DMEM: Dulbecco's modified Eagle's medium; FBS: Fetal bovine serum; PBS: Phosphate-buffered saline; CCK-8: Cell Counting Kit-8; GO: graphene oxide; PEG: polyethylene glycol; WB: Western blotting; FAC: Ferric ammonium citrate; T2WI: T2-weighted imaging; TR: Time of repetition; TE: Time of echo; FOV: Field of view; TEM: Transmission electron microscopy

\section{Declarations}

\section{Ethics approval and consent to participate}

All animal protocols used in this study were reviewed and approved by the Animal Care and Use Committee of Chongqing Medical University, and the experimental procedures were performed in accordance with the National Institutes of Health guidelines. 


\section{Consent for publication}

No applicable

\section{Availability of data and materials}

The datasets used and/or analyzed during the current study are available from the corresponding author on reasonable request.

\section{Competing interests}

The authors declare that they have no competing interests.

\section{Funding}

This study was supported by a grant from the National Natural Science Foundation of China (no. 81771892).

\section{Authors' contributions}

GB and JC supervised the research. All authors were involved in the design of the study or the acquisition and analysis of the data. GB, JS, JH, HZ, JW, YF, XZ and BX performed related experiments. GB, JS, HZ, JQ, JH, JW and YF analyzed the data and prepared the figures. GB, JS and JC wrote the main manuscript. GB and JS contributed equally to this work. All authors read and approved the final manuscript.

\section{Acknowledgments}

We are grateful to the Key Laboratory of Pediatrics in Chongqing for providing parts of the experimental apparatus. We also thank American Journal Experts (AJE) for editing the English language in our manuscript.

\section{References}

1. Lu TX, Rothenberg ME. MicroRNA. J Allergy Clin Immunol. 2018;141:1202-7.

2. Liu Z, Zhang XH, Callejas-Díaz B, Mullol J. MicroRNA in united airway diseases. Int J Mol Sci. 2016;17:716.

3. Ha M, Kim VN. Regulation of microRNA biogenesis. Nat Rev Mol Cell Biol. 2014;15:509-24.

4. Bandrés E, Cubedo E, Agirre X, Malumbres R, Zárate R, Ramirez N, et al. Identification by real-time PCR of 13 mature microRNAs differentially expressed in colorectal cancer and non-tumoral tissues. Mol Cancer. 2006;5:29.

5. Bommer GT, Gerin I, Feng Y, Kaczorowski AJ, Kuick R, Love RE, et al. p53-mediated activation of miRNA34 candidate tumor-suppressor genes. Curr Biol. 2007;17:1298-307.

6. Si ML, Zhu S, Wu H, Lu Z, Wu F, Mo YY. miR-21-mediated tumor growth. Oncogene. 2007;26:2799-803.

7. Asangani IA, Rasheed SA, Nikolova DA, Leupold JH, Colburn NH, Post S, et al. MicroRNA-21 (miR-21) posttranscriptionally downregulates tumor suppressor Pdcd4 and stimulates invasion, intravasation and metastasis in colorectal cancer. Oncogene. 2008;27:2128-36.

8. Du J, Yang S, An D, Hu F, Yuan W, Zhai C, et al. BMP-6 inhibits microRNA-21 expression in breast cancer through repressing deltaEF1 and AP-1. Cell Res. 2009;19:487-96. 
9. Markou A, Tsaroucha EG, Kaklamanis L, Fotinou M, Georgoulias V, Lianidou ES. Prognostic value of mature microRNA-21 and microRNA-205 overexpression in non-small cell lung cancer by quantitative real-time RTPCR. Clin Chem. 2008;54:1696-704.

10. Li T, Li D, Sha J, Sun P, Huang Y. MicroRNA-21 directly targets MARCKS and promotes apoptosis resistance and invasion in prostate cancer cells. Biochem Biophys Res Commun. 2009;383:280-5.

11. Gao JS, Zhang Y, Tang X, Tucker LD, Tarwater PM, Quesenberry PJ, et al. The evi1, microRNA-143, K-Ras axis in colon cancer. FEBS Lett. 2011;585:693-9.

12. Yao Q, Cao Z, Tu C, Zhao Y, Liu H, Zhang S. MicroRNA-146a acts as a metastasis suppressor in gastric cancer by targeting WASF2. Cancer Lett. 2013;335:219-24.

13. Cheng W, Liu T, Wan X, Gao Y, Wang H. MicroRNA-199a targets CD44 to suppress the tumorigenicity and multidrug resistance of ovarian cancer-initiating cells. Febs j. 2012;279:2047-59.

14. Chen Y, Tsai YH, Fang Y, Tseng SH. Micro-RNA-21 regulates the sensitivity to cisplatin in human neuroblastoma cells. J Pediatr Surg. 2012;47:1797-805.

15. Cheng AM, Byrom MW, Shelton J, Ford LP. Antisense inhibition of human miRNAs and indications for an involvement of miRNA in cell growth and apoptosis. Nucleic Acids Res. 2005;33:1290-7.

16. Sun X, Chen Y, Yu H, Machan JT, Alladin A, Ramirez J, et al. Anti-miRNA oligonucleotide therapy for chondrosarcoma. Mol Cancer Ther. 2019;18:2021-9.

17. Pall GS, Codony-Servat C, Byrne J, Ritchie L, Hamilton A. Carbodiimide-mediated cross-linking of RNA to nylon membranes improves the detection of siRNA, miRNA and piRNA by northern blot. Nucleic Acids Res. 2007;35:e60.

18. Zhang L, Wei P, Shen X, Zhang Y, Xu B, Zhou J, et al. MicroRNA expression profile in penile cancer revealed by next-generation small RNA sequencing. PLoS One. 2015;10:e0131336.

19. Thomson JM, Parker J, Perou CM, Hammond SM. A custom microarray platform for analysis of microRNA gene expression. Nat Methods. 2004;1:47-53.

20. Zhou Z, Lu ZR. Molecular imaging of the tumor microenvironment. Adv Drug Deliv Rev. 2017;113:24-48.

21. Mezzanotte L, van 't Root $M$, Karatas H, Goun EA, Löwik C. In vivo molecular bioluminescence imaging: new tools and applications. Trends Biotechnol. 2017;35:640-52.

22. Lu CY, Ji JS, Zhu XL, Tang PF, Zhang Q, Zhang NN, et al. T2-weighted magnetic resonance imaging of hepatic tumor guided by SPIO-loaded nanostructured lipid carriers and ferritin reporter genes. ACS Appl Mater Interfaces. 2017;9:35548-61.

23. Li D, Li Y. The interaction between ferroptosis and lipid metabolism in cancer. Signal Transduct Target Ther. 2020;5:108.

24. He X, Cai J, Li H, Liu B, Qin Y, Zhong Y, et al. In vivo magnetic resonance imaging of xenografted tumors using FTH1 reporter gene expression controlled by a tet-on switch. Oncotarget. 2016;7:78591-604.

25. Iordanova B, Ahrens ET. In vivo magnetic resonance imaging of ferritin-based reporter visualizes native neuroblast migration. Neuroimage. 2012;59:1004-12.

26. Ziv K, Meir G, Harmelin A, Shimoni E, Klein E, Neeman M. Ferritin as a reporter gene for MRI: chronic liver over expression of $\mathrm{H}$-ferritin during dietary iron supplementation and aging. NMR Biomed. 2010;23:523-31.

27. Velde GV, Rangarajan JR, Toelen J, Dresselaers T, Ibrahimi A, Krylychkina O, et al. Evaluation of the specificity and sensitivity of ferritin as an MRI reporter gene in the mouse brain using lentiviral and adeno- 
associated viral vectors. Gene Ther. 2011;18:594-605.

28. Cohen B, Ziv K, Plaks V, Harmelin A, Neeman M. Ferritin nanoparticles as magnetic resonance reporter gene. Wiley Interdiscip Rev Nanomed Nanobiotechnol. 2009;1:181-8.

29. Mu T, Qin Y, Liu B, He X, Liao Y, Sun J, et al. In vitro neural differentiation of bone marrow mesenchymal stem cells carrying the FTH1 reporter gene and detection with MRI. Biomed Res Int. 2018;2018:1978602.

30. Zhang M, Liu X, Huang J, Wang L, Shen H, Luo Y, et al. Ultrasmall graphene oxide based T(1) MRI contrast agent for in vitro and in vivo labeling of human mesenchymal stem cells. Nanomedicine. 2018;14:2475-83.

31. Gu Y, Guo Y, Wang C, Xu J, Wu J, Kirk TB, et al. A polyamidoamne dendrimer functionalized graphene oxide for DOX and MMP-9 shRNA plasmid co-delivery. Mater Sci Eng C Mater Biol Appl. 2017;70:572-85.

32. Wang F, Zhang B, Zhou L, Shi Y, Li Z, Xia Y, et al. Imaging dendrimer-grafted graphene oxide mediated antimiR-21 delivery with an activatable luciferase reporter. ACS Appl Mater Interfaces. 2016;8:9014-21.

33. Ribeiro BFM, Souza MM, Fernandes DS, do Carmo DR, Machado-Santelli GM. Graphene oxide-based nanomaterial interaction with human breast cancer cells. J Biomed Mater Res A. 2020;108:863-70.

34. Qu Y, Sun F, He F, Yu C, Lv J, Zhang Q, et al. Glycyrrhetinic acid-modified graphene oxide mediated siRNA delivery for enhanced liver-cancer targeting therapy. Eur J Pharm Sci. 2019;139:105036.

35. Alibolandi M, Mohammadi M, Taghdisi SM, Ramezani M, Abnous K. Fabrication of aptamer decorated dextran coated nano-graphene oxide for targeted drug delivery. Carbohydr Polym. 2017;155:218-29.

36. Sun J, Huang J, Bao G, Zheng H, Wang C, Wei J, et al. MRI detection of the malignant transformation of stem cells through reporter gene expression driven by a tumor-specific promoter. Stem Cell Res Ther. $2021 ; 12: 284$.

37. Kim S, Hwang DW, Lee DS. A study of microRNAs in silico and in vivo: bioimaging of microRNA biogenesis and regulation. FEBS J. 2009;276:2165-74.

38. Ko HY, Hwang DW, Lee DS, Kim S. A reporter gene imaging system for monitoring microRNA biogenesis. Nat Protoc. 2009;4:1663-9.

39. Kim HJ, Chung JK, Hwang DW, Lee DS, Kim S. In vivo imaging of miR-221 biogenesis in papillary thyroid carcinoma. Mol Imaging Biol. 2009;11:71-8.

40. Song H, Oh B, Choi M, Oh J, Lee M. Delivery of anti-microRNA-21 antisense-oligodeoxynucleotide using amphiphilic peptides for glioblastoma gene therapy. J Drug Target. 2015;23:360-70.

41. Costa PM, de Lima MCP. MicroRNAs as molecular targets for cancer therapy: on the modulation of MicroRNA expression. Pharmaceuticals (Basel). 2013;6:1195-220.

42. Liu XQ, Song WJ, Sun TM, Zhang PZ, Wang J. Targeted delivery of antisense inhibitor of miRNA for antiangiogenesis therapy using CRGD-functionalized nanoparticles. Mol Pharm. 2011;8:250-9.

\section{Figures}




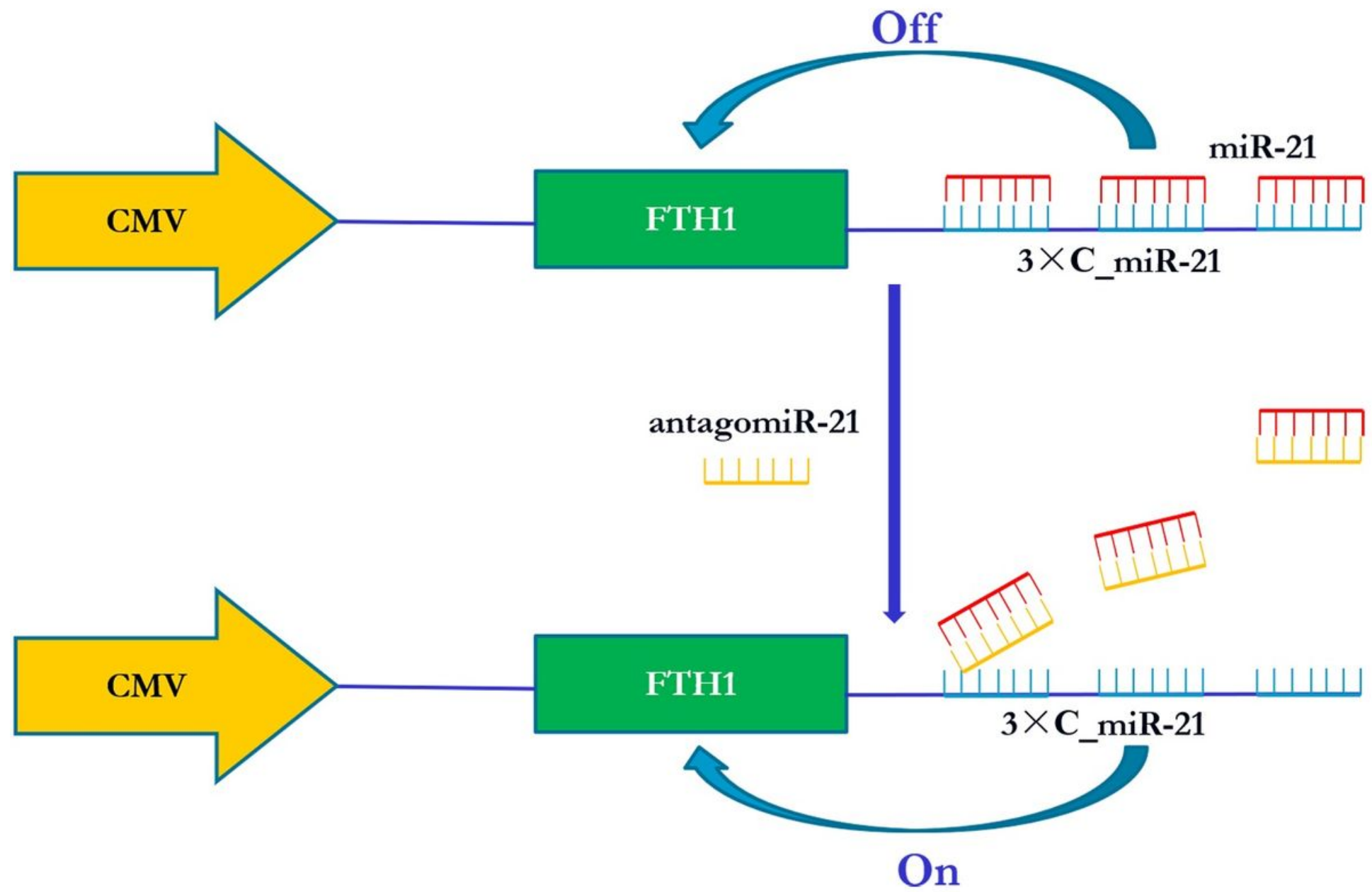

Figure 1

Schematic illustration of the miR-21-responsive FTH1 gene reporter system. The reporter gene FTH1 is modified by the addition of 3 copies of the sequence completely complementary to miR-21 (3xC_miR-21) to its 3'untranslated region (3' UTR). In the presence of miR-21, FTH1 expression is switched "off" due to the combination of miR-21 with the 3' UTR of the reporter gene. Once an exogenous antisense oligonucleotide (antagomiR-21) is administered, it binds to miR-21 in a competitive manner and releases miR-21 from the 3' UTR of FTH1, thereby switching "on" reporter gene expression. 

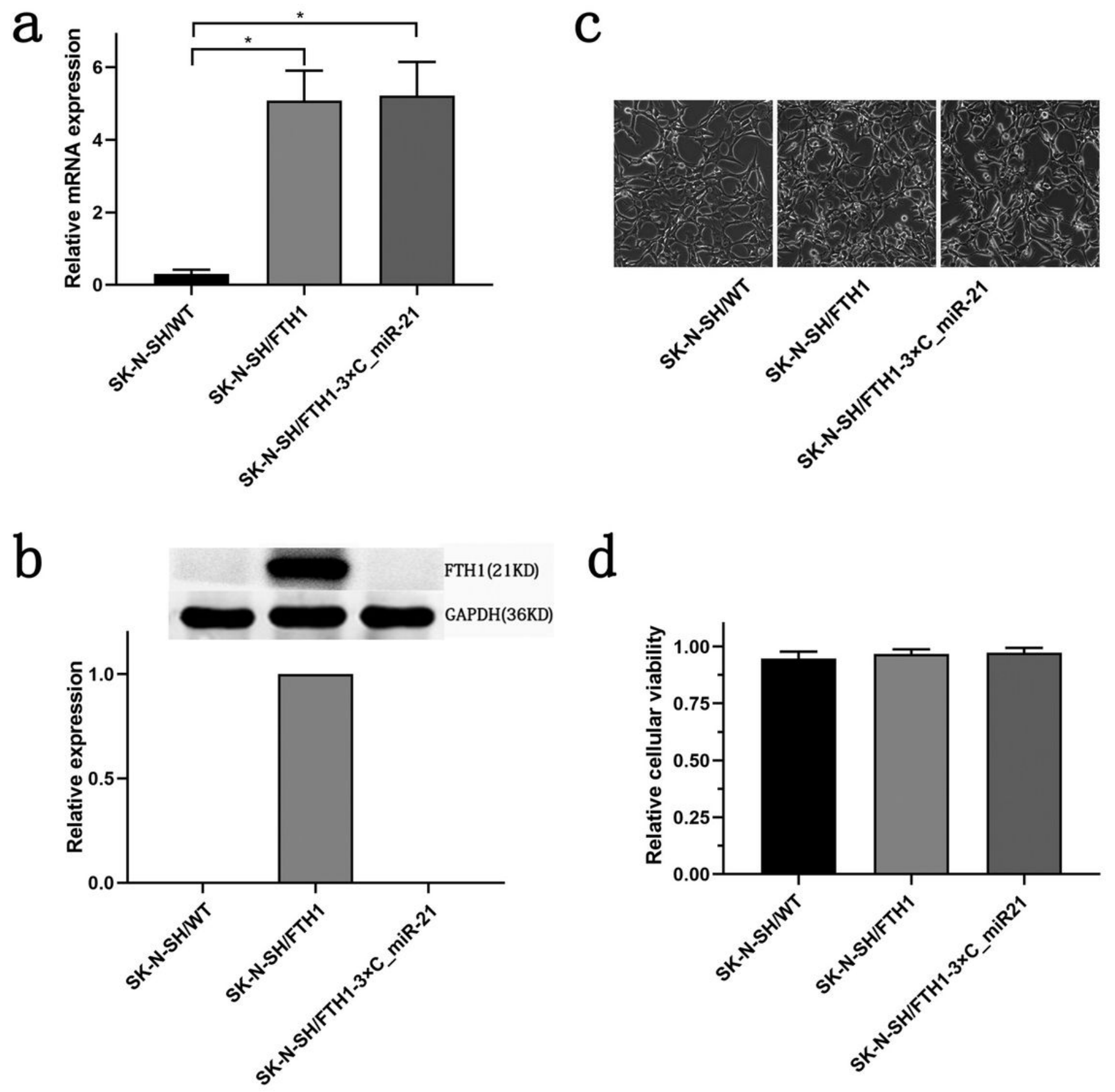

Figure 2

FTH1 expression and its effect on viability in the three groups of cells. The qRT-PCR results (a) showed that the mRNA expression levels of the FTH1 gene in the SK-N-SH/FTH1 and SK-N-SH/FTH1-3 × C_miR-21 groups was similar, and both of them had significantly higher levels than the SK-N-SH/ WT group. The WB results (b) showed that compared with the SK-N-SH/WT group, the SK-N-SH/FTH1 group showed significantly increased FTH1 expression at the protein level $(\mathrm{P}<0.0001)$, while the SK-N-SH/FTH1-3×C_miR-21 group did not show alterations in FTH1 expression. No morphological changes were observed among the SK-N-SH/WT, SK-N-SH/FTH1 and SKN-SH/FTH1-3×C_miR-21 cells (c). The CCK-8 results revealed no difference in cell viability among the three groups (d). ${ }^{\star \star \star \star} P<0.0001$. 


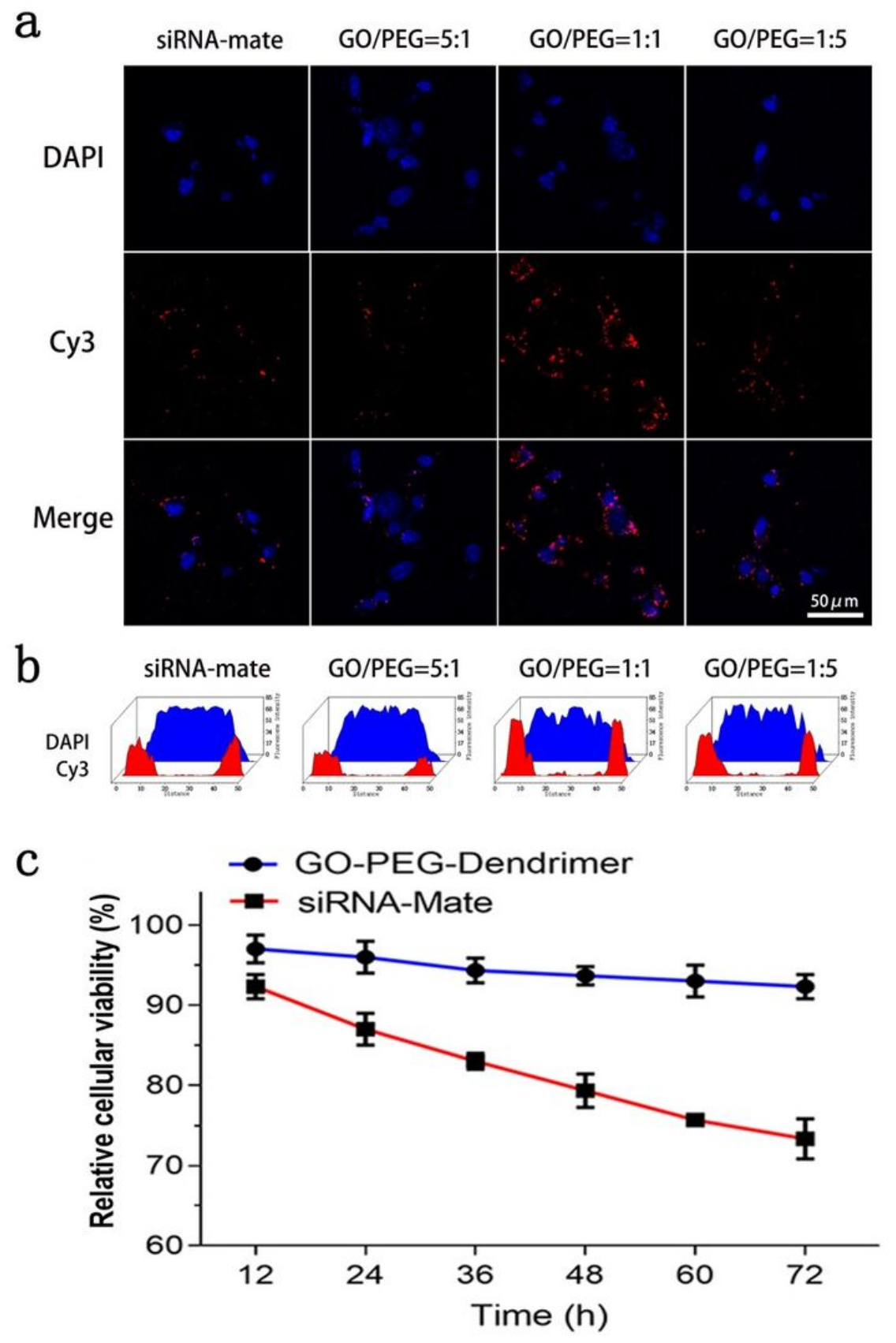

\section{Figure 3}

Transfection efficiency and cytotoxicity of the GO-PEG-dendrimer. Both the immunofluorescence staining (a) and fluorescence intensity quantification (b) results show that among the three GO-PEG-dendrimer structures with different GO/PEG ratios, the structure with a GO/PEG ratio of 1:1 had the highest transfection efficiency and was more efficient than the commonly used transfection reagent, siRNA-Mate. The other two structures with GO/PEG ratios of 5:1 and 1:5 had transfection efficiencies that were similar to that of the siRNA-Mate. The CCK-8 assay (c) showed that the GO-PEG-dendrimer was much less cytotoxic than siRNA-Mate during the $72 \mathrm{~h}$ of coculture with SK-N-SH cells. 
a

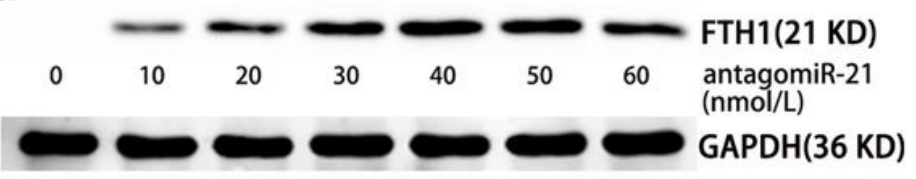

b

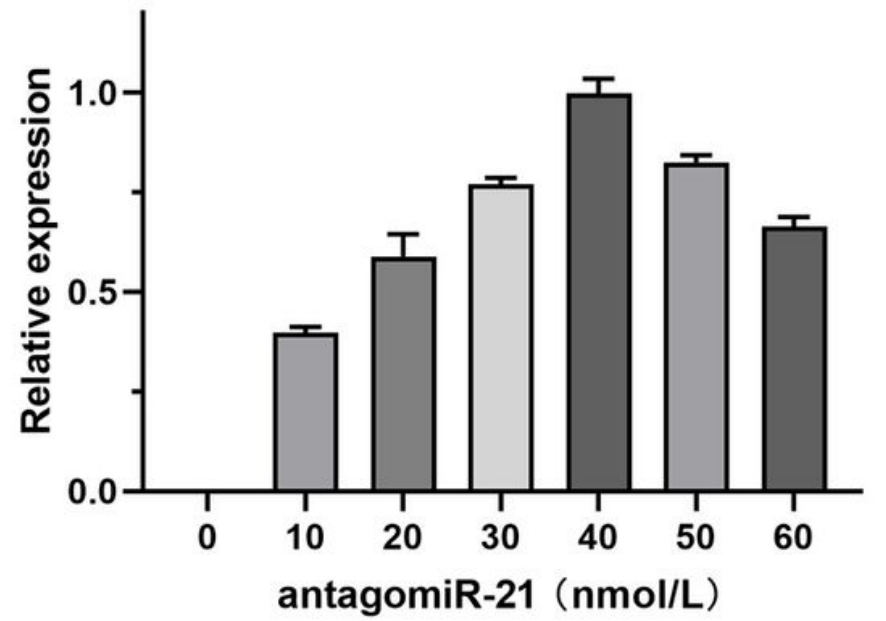

C

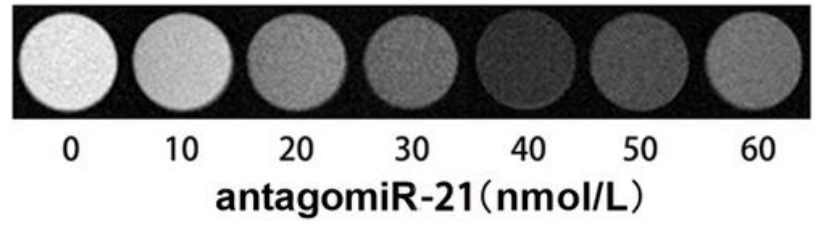

d

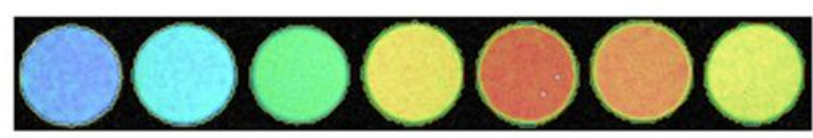

$\begin{array}{llllll}0 & 10 & 20 & 30 & 40 & 50\end{array}$

60
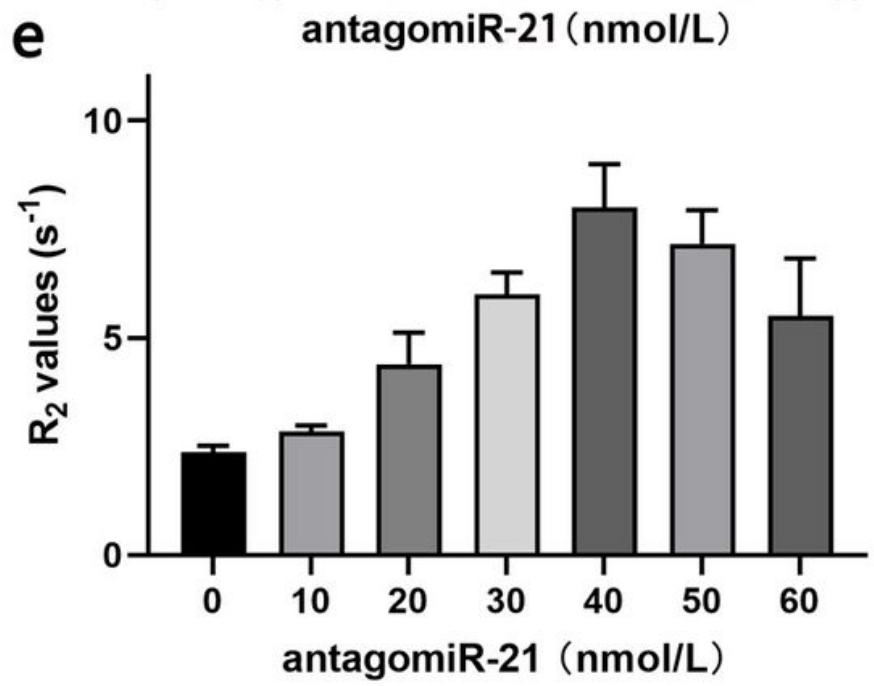

Figure 4

Regulation of FTH1 expression by antagomiR-21. WB (a) and gray-scale (b) results show that the delivery of antagomiR-21 into cells reversed FTH1 expression in a dose-dependent manner. In the absence of antagomiR-21, no obvious FTH1 expression was detected in SK-N-SH/FTH1-3 $\times$ PT cells in which miR-21 suppressed the reporter gene by binding with the completely complimentary sequence of 3×C_miR-21. After transfection with antagomir-21, the expression of FTH1 increased gradually as the antagomir-21 concentration increased, peaking at $40 \mathrm{nmol} / \mathrm{L}$, and then slightly decreased as the antagomiR-21 concentration continuously increased. The cellular MRI T2WI (c), T2 map (d) and R2 measurements (e) in cells treated with different concentrations of antagomiR-21 were consistent with the WB results. 


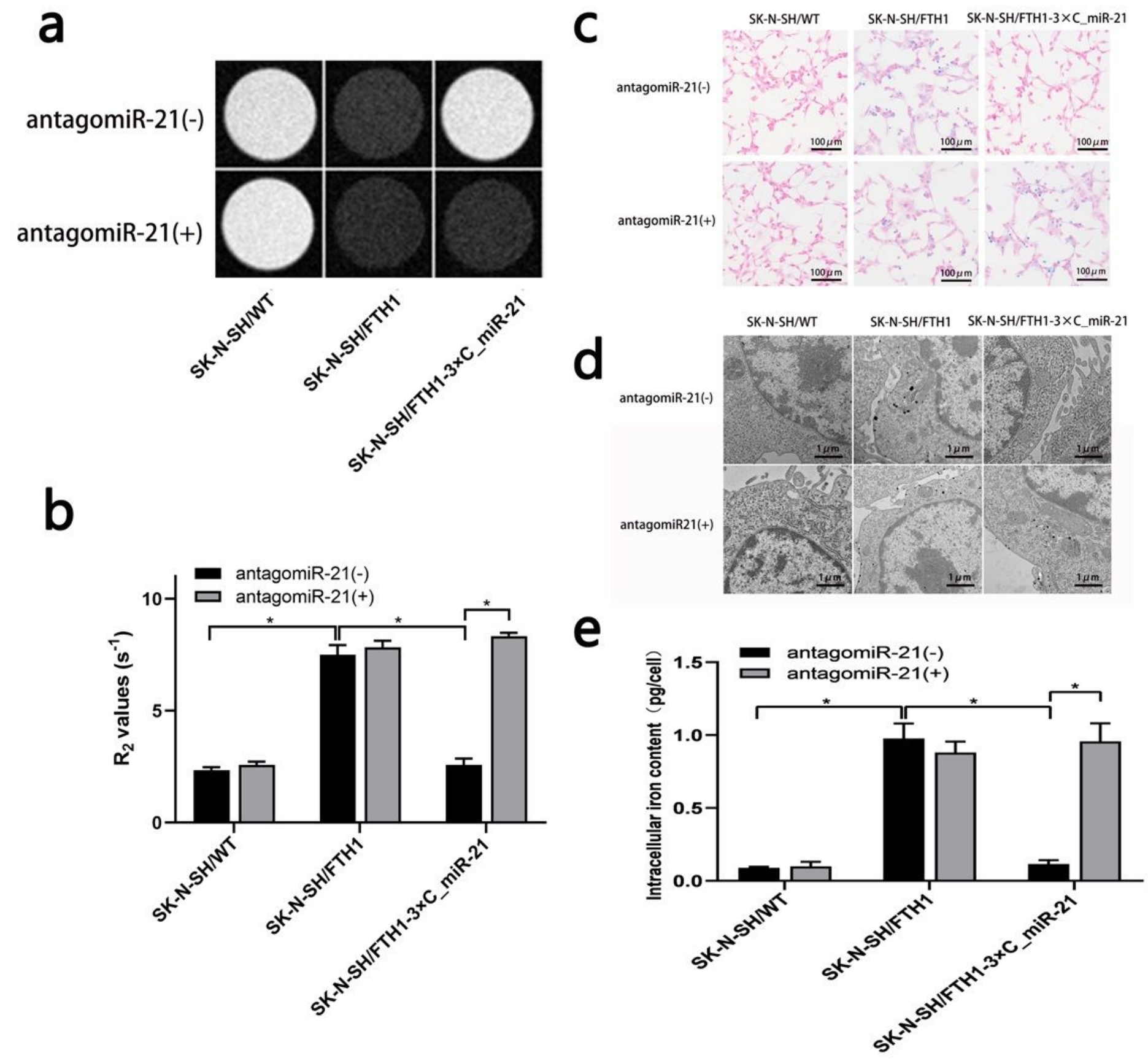

Figure 5

Cellular MRI and intracellular iron detection. MRI imaging shows a significant T2WI signal reduction (a) and R2 value increase (b) in the SK-N-SH/FTH1-3×C_miR-21 cells after antagomiR-21 transfection compared with that prior to antagomiR-21 treatment. In the SK-N-SH/WT and SK-N-SH/FTH1 cells, however, there were no differences in the MRI signals or R2 values before and after antagomiR-21 treatment. The Prussian blue staining (c) and TEM (d) results revealed that before antagomiR-21 transfection, large amounts of iron particles were detected in the cytoplasm of only SK-N-SH/FTH1 cells, while iron accumulation was not observed in SK-NSH/WT or SK-N-SH/FTH1-3×C_miR-21 cells. After antagomiR-21 treatment, SK-N-SH/FTH1-3×C_miR-21 cells exhibited obvious iron accumulation compared with that prior to antagomiR-21 treatment. In the other two groups of cells, however, there was almost no change in the iron accumulation before and after antagomiR-21 
treatment. Further iron content quantification (e) shows the same results as observed in Prussian blue staining and TEM. * $\mathrm{P}<0.05$.

a

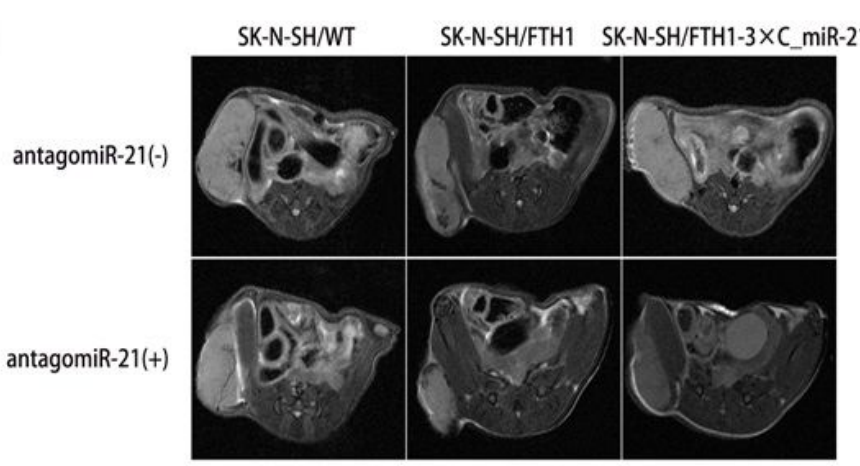

b

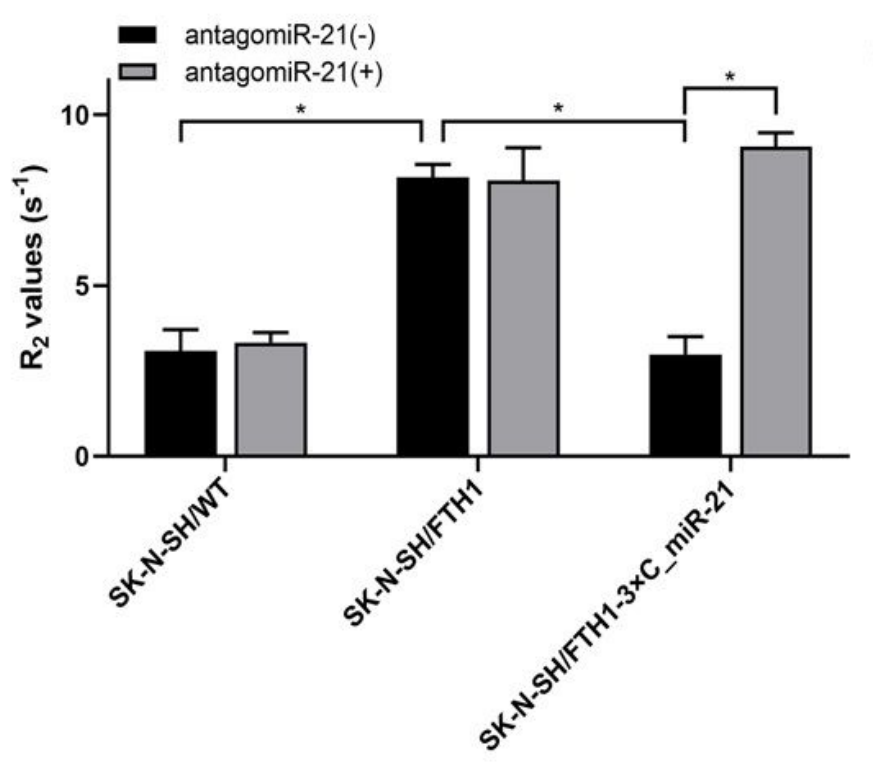

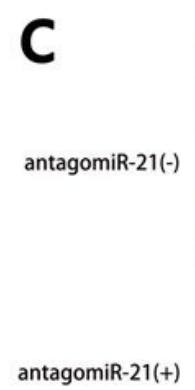

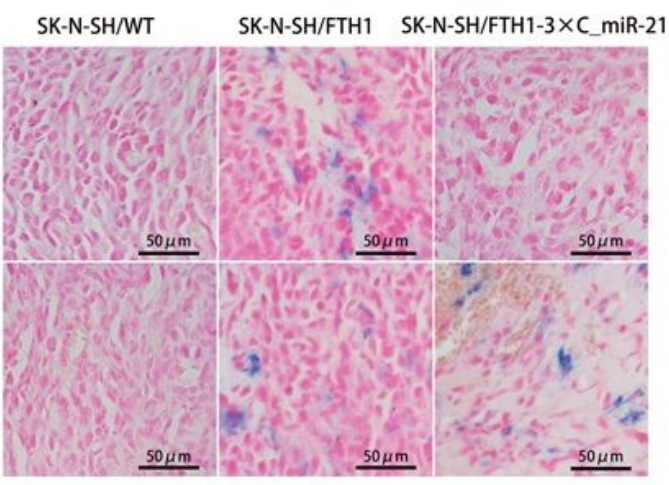

d

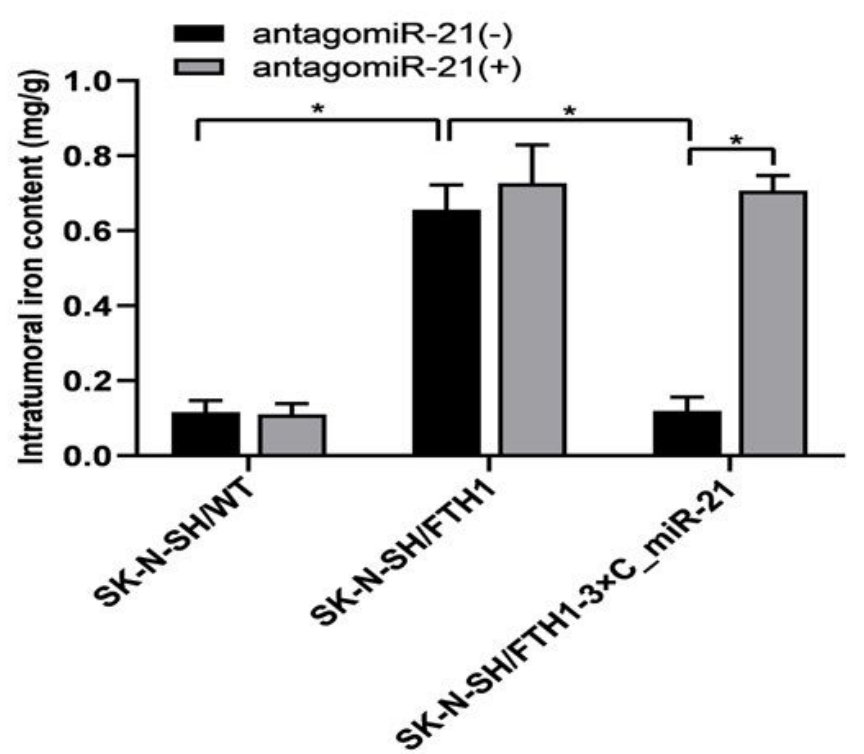

Figure 6

$\mathrm{MRI}$ and iron detection in xenografts before and after antagomiR-21 administration. Before antagomiR-21 administration, the MRI signal in the SK-N-SH/FTH1 group was obviously decreased and the R2 value was significantly increased compared with those in the SK-N-SH/WT group, but these values in the SK-N-SH/FTH1$3 \times$ C_miR-21 group did not differ from those in the SK-N-SH/WT group. After treatment with the antagomiR-21, the SK-N-SH/FTH1-3×C_miR-21 group showed a significantly decreased MRI signal and increased R2 value compared with those prior to treatment. In the other two groups, the MRI signals and R2 values did not differ before and after antagomiR-21 treatment ( $a$ and $b$ ). The Prussian blue staining (c) and intratumoral iron content quantification (d) results match the MRI results. Intratumoral iron accumulation was significantly increased after antagomiR-21 treatment in only the SK-N-SH/FTH1-3×C_miR-21 group. * $\mathrm{P}<0.05$.

\section{Supplementary Files}

This is a list of supplementary files associated with this preprint. Click to download.

- Supplementaryinformation.docx 